EPJ manuscript No.

(will be inserted by the editor)

\title{
Use of the chemical potential for a limited number of fermions with a degenerate groundstate
}

\author{
L.F. Lemmens ${ }^{1}$, F. Brosens ${ }^{2}$, and J.T. Devreese ${ }^{2}$ \\ ' 1 Departement Natuurkunde, Universiteit Antwerpen (RUCA), \\ Groenenborgerlaan 171, B-2020 Antwerpen, Belgium. \\ 2 Departement Natuurkunde, Universiteit Antwerpen (UIA), \\ Universiteitsplein 1, B-2610 Antwerpen, Belgium, \\ Received: date / Revised version: date
}

\begin{abstract}
For fermions with degenerate single-particle energy levels, the usual relation between the total number of particles and the chemical potential $\mu$ is only satisfied for a specific number of particles, i.e. those leading to closed shells. The treatment of an arbitrary number of fermions requires a modification of the chemical potential, similar to the one proposed by Landsberg for Bose-condensed systems. We study the implications of the required modification for fermions in a potential, by calculating the ground state energy, the free energy, the density, the partition function and the dynamic two-point correlation function. It turns out that the modified relation between the fugacity and the number of particles leads to the correct ground state energy and density. But for other quantities like the entropy and the two-point correlation functions, an additional correction is required and derived. These calculations indicate that many-body perturbation theories based on $H-\mu N$ with Lagrange multiplier $\mu$, are not applicable in unmodified form for a fixed number of fermions at low temperature.
\end{abstract}

PACS. 05.30.Fk Fermion systems and electron gas - 03.75.Ss Degenerate Fermi gases

\section{Introduction}

When studying many-body effects in systems with Hamiltonian $H$ for a finite number $N$ of particles, it is often convenient to introduce the chemical potential $\mu$ as a Lagrange multiplier associated with $N$ [1,2,3, and to inves' tigate properties related to $H-\mu N$. E.g., the Gibbs free energy $G$ is obtained from $e^{-\beta G}=\operatorname{Tr} e^{-\beta(H-\mu N)}$, where the trace is taken over all the degrees of freedom, including the number of particles. After the calculations, the chemical potential is then determined from the constraint that the number of particles under consideration be $N$, e.g., to obtain the free energy $F$ from $e^{-\beta F}=\operatorname{Tr} e^{-\beta H}$, where the latter trace is taken over the degrees of freedom of the $N$ particles, with $N$ fixed. This procedure can provide a very efficient way to deal with the finite particle-number, but it has to be handled with care [4, because in unmodified form it is only valid for closed shell systems.

We show that a modified expression for the fugacity $u$ of the form $u=\lambda e^{\mu \beta}$, as introduced by Landsberg [5] in the case of Bose condensed systems, instead of the usual expression $e^{\mu \beta}$, provides a relation between the total number of particles and the fugacity for any number of particles, also if the ground state is degenerate. It leads to the correct ground state energy and density. But even the use of the modified constraint on the number of particles does not provide correct results for other quantities. E.g. the calculations of the entropy and of the two-point correlation functions require specific and well-defined corrections, to be derived below.

Our derivation is based on the $\mathrm{N}$-particle density matrix, properly anti-symmetrized for fermions. With the aid of this quantity we can express the partition function, the density and the density-density correlation function in terms of single-particle density matrices. The method allows for the analytical calculation of the partition function and the correlation functions in the low temperature limit. We show explicitly how the partition function and the density can be calculated in order to elucidate the same method used in the more involved calculation of the density-density correlation function. In essence, the method proceeds as follows. The anti-symmetrization induces a summation over all possible cycles of the permutation group. In order to avoid the difficult constraint on the cycle lengths, arising from the fixed number of particles, a generating function is constructed by forming a power series in $u$ with the elements $A_{N}$ of the sequence $\left\{A_{N} \mid N=1, \ldots, \infty\right\}$ as coefficients, where $A_{N}$ is the result of the cyclic summation for $N$ particles. This method is standard in quantum statistical mechanics 6 7, $8,9,10$, and very useful to construct path integrals for identical particles 11,12 13,14]. 15. Replacing $u$ by $\frac{1}{z}$ it is indicated 
as the $Z$-transform:

$$
\widetilde{A}(z)=\sum_{n=0}^{\infty} A_{n} \frac{1}{z^{n}}
$$

Once the generating functions are known we have to expand them in a power series in $u$, and extract the coefficient of the $N^{t h}$ power of $u$ to obtain the expression corresponding with a fermion gas of $N$ particles [16]. Using this technique the partition function, the one-point and two-point correlation function can be obtained directly i.e. without the use of any quantity related to the chemical potential.

It should be noted that we could easily have advertised this investigation under the common denominator of "non-equivalence between ensembles" [17,18 19,20,21, [22 23 24 25] 26 27. But we refrain from doing so for the following reason. Starting from a stationary Hamiltonian with a countable spectrum, one can construct a density of states for a given total energy and a given number of particles. The partition function is the Laplace transform of this density of states with respect to the energy, and the grand canonical partition function is the generating function of this partition function with respect to number of particles. In this respect the three functions only describe the same information in different parameter spaces

$$
\{E, N\} \underbrace{\Longleftrightarrow}_{\text {Laplace-transform }}\{\beta, N\} \underbrace{\Longleftrightarrow}_{\text {Z-transform }}\{\beta, u\} .
$$

Only if the transforms are approximately evaluated, the warranty that they lead to the same results expires [28]. This approach is based on the fundamental transforms between $\Omega, Z$ and $\Xi$, and it indicates that the calculation of properties of a system with a finite number of particles using the chemical potential - even with the modification - is an approximation as soon as the levels of the Fermisystem are degenerate.

In the next section we introduce the basic methods, and illustrate them by analyzing the partition function. In the subsequent sections, the same methodology is used to study the one-point and the two-point correlation functions. Finally, we will discuss our results and their implications for the $N$-body problem.

\section{The partition function of an open shell fermion gas}

In this section we will elucidate the mathematical methods used throughout the paper, by analyzing the partition function of an open shell fermion gas as an example. It is well known that the Fermi-Dirac distribution can be derived from the probabilistic problem of distributing $N$ fermions on a given set of states, assuming that a state can contain at most one fermion. The resulting distribution is easily derived using generating functions 29]. Assume that the energy of a state is given by $\epsilon_{\nu}$. The energy $E$ of the ground state (i.e., the total energy of all occupied states at zero temperature) can then be calculated. In the case of a one-dimensional (1D) oscillator with frequency $\omega$ the energy spectrum is given by $\epsilon_{\nu}=\hbar \omega\left(\nu+\frac{1}{2}\right)$, with degeneracy $g_{\nu}=1$. Placing $N$ fermions in the system leads to the ground state energy $E=\hbar \omega \sum_{\nu=0}^{N-1}\left(\nu+\frac{1}{2}\right)$. The occupancy density $p_{\nu}$ of a state expressed in terms of its energy becomes $p_{\nu}=\frac{1}{N} \Theta\left(\epsilon_{\nu}<\epsilon_{N}\right)$, where $\epsilon_{N}$ is the lowest energy of the unoccupied states. The generalized Heaviside function used here is defined as

$$
\Theta(x)=\left\{\begin{array}{l}
1 \text { if } \mathrm{x} \text { is true } \\
0 \text { otherwise }
\end{array}\right.
$$

However, the non-degenerate case is the exception rather than the rule. We might also consider an illustration of the case of open shells. E.g., for a two-dimensional (2D) oscillator, the number of states with the same energy $\epsilon_{\nu}$ is characterized by the degeneracy $g_{\nu}=\nu+1$, whereas for the 3D oscillator $g_{\nu}=(\nu+1)(\nu+2) / 2$. The set of states with the same energy $\epsilon_{\nu}$ is called a level or a shell, labeled by the (nonnegative integer) index $\nu$.

If $L$ denotes the index of the lowest not completely occupied shell (i.e., either empty or partially filled), then the number $N_{L}$ of fermions accommodated in completely filled shells is $N_{L}=\sum_{\nu=0}^{L-1} g_{\nu}$. If not all shells are completely filled, there are $N-N_{L}$ fermions left over to occupy the shell with index $L$. The ground state energy is therefore

$$
E_{0}(N)=\sum_{\nu=0}^{L-1} g_{\nu} \epsilon_{\nu}+\left(N-N_{L}\right) \epsilon_{L}
$$

The energy difference at low temperature between a gas containing $N$ particles and one containing $(N-1)$ particles is given by:

$$
E_{0}(N)-E_{0}(N-1)=\epsilon_{L},
$$

provided that the gas with $N$ particles is an open shell gas. In textbooks this difference is sometimes used as the definition of the chemical potential. In the present paper, we follow the convention that $\mu$ is the parameter that is used to satisfy the constraint arising of the finite number of particles as discussed in the introduction.

\subsection{From density of states to generating function and vice versa}

The local density of states matrix $\Omega\left(E, \mathbf{r}^{\prime}, \mathbf{r}\right)$ for a particle in a one-body potential can be defined as

$$
\Omega\left(E, \mathbf{r}^{\prime}, \mathbf{r}\right)=\sum_{\nu=0}^{\infty} \delta\left(E-\epsilon_{\nu}\right) \sum_{k=1}^{g_{\nu}} \varphi_{\nu, k}\left(\mathbf{r}^{\prime}\right) \varphi_{\nu, k}^{*}(\mathbf{r}),
$$

where $\varphi_{\nu, k}(\mathbf{r})$ are ortho-normal wave functions with corresponding eigenvalues $\epsilon_{\nu}$. The index $\nu$ accounts for the energy levels and the index $k$ runs from 1 to $g_{\nu}$, where $g_{\nu}$ 
is the degeneracy of the energy level $\epsilon_{\nu}$. The diagonal part of this matrix is given by

$$
\Omega(E, \mathbf{r})=\sum_{\nu=0}^{\infty} \delta\left(E-\epsilon_{\nu}\right) \sum_{k=1}^{g_{\nu}}\left|\varphi_{\nu, k}(\mathbf{r})\right|^{2},
$$

and the density of states $\Omega(E)$ becomes

$$
\Omega(E) \equiv \int \Omega(E, \mathbf{r}) d \mathbf{r}=\sum_{\nu=0}^{\infty} g_{\nu} \delta\left(E-\epsilon_{\nu}\right)
$$

The single-particle propagator in its spectral representation is usually written as

$$
K_{1}\left(\mathbf{r}^{\prime}, \beta \mid \mathbf{r}, 0\right)=\sum_{\nu=0}^{\infty} e^{-\beta \epsilon_{\nu}} \sum_{k=1}^{g_{\nu}} \varphi_{\nu, k}\left(\mathbf{r}^{\prime}\right) \varphi_{\nu, k}^{*}(\mathbf{r})
$$

and it is clearly the Laplace transform of $\Omega\left(E, \mathbf{r}^{\prime}, \mathbf{r}\right)$

$$
K_{1}\left(\mathbf{r}^{\prime}, \beta \mid \mathbf{r}, 0\right)=\int \Omega\left(E, \mathbf{r}^{\prime}, \mathbf{r}\right) e^{-\beta E} d E,
$$

with $\beta$ adjoined to $E$, provided the Laplace transform exists, i.e., that the energy levels $\epsilon_{\nu}$ are positive. This means that the origin of the energy spectrum is chosen such that it does not exceed the lowest eigen-energy $\epsilon_{0}$. The relation between the single-particle partition function $Z_{1}(\beta)$ and the single-particle propagator

$$
Z_{1}(\beta)=\iint K_{1}\left(\mathbf{r}^{\prime}, \beta \mid \mathbf{r}, 0\right) \delta\left(\mathbf{r}^{\prime}-\mathbf{r}\right) d \mathbf{r} d \mathbf{r}^{\prime}
$$

implies that the single-particle partition function can also be written as a Laplace transform of the density of states:

$$
Z_{1}(\beta)=\sum_{\nu=0}^{\infty} g_{\nu} e^{-\beta \epsilon_{\nu}}=\int \Omega(E) e^{-\beta E} d E
$$

with the inverse relation given by

$$
\Omega(E)=\frac{1}{2 \pi i} \oint_{c} Z_{1}(s) e^{s E} d s
$$

where $c$ is a Bromwich contour.

The propagator $\mathcal{K}_{D}$ of $N$ distinguishable particles with position vectors $\mathbf{r}_{j}(j=1, \ldots, N)$, all in the same potential, is the product of their single-particle propagators

$$
\mathcal{K}_{D}\left(\bar{r}, \beta ; \bar{r}^{\prime}, 0\right)=\prod_{j=1}^{N} K_{1}\left(\mathbf{r}_{j}^{\prime}, \beta \mid \mathbf{r}_{j}, 0\right)
$$

The vector $\bar{r}$ denotes the configuration $\left(\mathbf{r}_{1}, \ldots, \mathbf{r}_{N}\right)^{T}$ of the $N$ particles in the system. When fermions in the same spin state are involved one has to anti-symmetrize this propagator

$$
\mathcal{K}_{F}\left(\bar{r}, \beta \mid \bar{r}^{\prime}, 0\right)=\frac{1}{N !} \sum_{P}(-1)^{P} \prod_{j=1}^{N} K_{1}\left(\{P \mathbf{r}\}_{j}, \beta \mid \mathbf{r}_{j}^{\prime}, 0\right) .
$$

The summation runs over all permutations $P$ of the particle indices, and the sign factor $(-1)^{P}$ assures that odd permutations contribute with a negative sign. For convenience in the notations, we limit the treatment to the spin-polarized case. The non-polarized case can be treated along the same lines, see e.g. 30]. is

The partition function for this system of $N$ fermions

$$
\mathcal{Z}_{F}(\beta \mid N)=\iint \mathcal{K}_{F}\left(\bar{r}, \beta \mid \bar{r}^{\prime}, 0\right) \delta\left(\bar{r}-\bar{r}^{\prime}\right) d \bar{r} d \bar{r}^{\prime}
$$

The integrals over the configuration space can be done using the decomposition of the permutations in cycles 6 . $7,8,9,10,31,32,33,34$. With $M_{\ell}$ denoting the number of cycles of length $\ell$, the partition function becomes

$$
\begin{aligned}
\mathcal{Z}_{F}(\beta \mid N)= & \sum_{M_{1} \cdots M_{N}} \Theta\left(\sum_{\ell=1}^{N} \ell M_{\ell}=N\right) \times \\
& \prod_{\ell=1}^{N} \frac{(-1)^{(\ell-1) M_{\ell}}}{M_{\ell} ! \ell^{M_{\ell}}}\left(Z_{1}(\ell \beta)\right)^{M_{\ell}} .
\end{aligned}
$$

Introducing a generating function

$$
\Xi_{F}(\beta, u)=\sum_{N=0}^{\infty} u^{N} \mathcal{Z}_{F}(\beta \mid N)
$$

the constraint $\sum_{\ell=1}^{N} \ell M_{\ell}=N$ can be removed, and the summation over $M_{\ell}$ can be done

$$
\Xi_{F}(\beta, u)=\exp \left(-\sum_{\ell=1}^{\infty} \frac{(-u)^{\ell}}{\ell} Z_{1}(\ell \beta)\right) .
$$

Using the spectral representation (8) of $Z_{1}(\beta)$, the sum over the cycles can easily be performed. The result is:

$$
\begin{aligned}
\Xi_{F}(\beta, u) & =\exp \left(\int \Omega(E) \ln \left(1+u e^{-\beta E}\right) d E\right) \\
& =\prod_{\nu=0}^{\infty}\left(1+u e^{-\beta \epsilon_{\nu}}\right)^{g_{\nu}} .
\end{aligned}
$$

Again as in the case of a single particle, the two variables $\beta, u$ have no specific physical meaning with respect to the parameters defining the system, i.e., the energy $E$ and the number of particles $N$. The partition function $\mathcal{Z}_{F}(\beta \mid N)$ for $N$ particles can then be obtained from its generating function $\Xi_{F}(\beta, u)$ by the inverse transformation

$$
\mathcal{Z}_{F}(\beta \mid N)=\left.\frac{1}{N !} \frac{d^{N} \Xi_{F}(\beta, u)}{d u^{N}}\right|_{u=0}
$$

Remark that in the derivation of (13) $u$ is nothing but an intermediate variable. If one substitutes $u$ by $1 / z$, one can state that $\Xi\left(\beta, \frac{1}{z}\right)$ is the $Z$ transform of the sequence

$$
\left\{1, \mathcal{Z}_{F}(\beta \mid 1), \ldots, \mathcal{Z}_{F}(\beta \mid N), \ldots\right\} \text {. }
$$


The inverse of the $Z$ transform is given by a contourintegration, leading to the following alternative inversion formula:

$$
\mathcal{Z}_{F}(\beta \mid N)=\frac{1}{2 \pi i} \oint_{C} \frac{\Xi_{F}(\beta, \gamma)}{\gamma^{N+1}} d \gamma
$$

where $C$ is a closed counterclockwise contour around the origin and chosen in such a way that only the origin is a singularity. The inversion can also be done using recursion formulas [35,36]. Although they are more appropriate for bosons, they are occasionally used for fermions 37.

\subsection{The inversion method and the chemical potential}

Since $\Xi_{F}(\beta, \gamma)$ is an analytic function of $\gamma$, a circular contour with radius $u$ can be considered, and introducing $\gamma=u e^{i \theta}$ the inversion integral can be rewritten as

$$
\mathcal{Z}_{F}(\beta \mid N)=\frac{1}{2 \pi} \int_{0}^{2 \pi} e^{\left[\ln \Xi_{F}\left(\beta, u e^{i \theta}\right)-N \ln u\right]} e^{-i N \theta} d \theta .
$$

Choosing the radius $u$ such that the argument

$$
\left[\ln \Xi_{F}\left(\beta, u e^{i \theta}\right)-N \ln u\right]
$$

of the exponential function reaches an extremum, one obtains the condition

$$
N=u \frac{d}{d u} \ln \Xi_{F}(\beta, u)=\int \Omega(E) \frac{u e^{-\beta E}}{1+u e^{-\beta E}} d E .
$$

Denoting the solution $u$ obtained from this implicit equation by $u_{e}$, the partition function can be rewritten as

$$
\begin{aligned}
\mathcal{Z}_{F}(\beta \mid N) & =\mathcal{Z}_{e}(\beta \mid N) \int_{0}^{2 \pi} \Psi(\theta) d \theta \\
\mathcal{Z}_{e}(\beta \mid N) & =\frac{\Xi\left(\beta, u_{e}\right)}{u_{e}} \\
\Psi(\theta) & =\frac{1}{2 \pi} \frac{\Xi\left(\beta, u_{e} e^{i \theta}\right)}{\Xi\left(\beta, u_{e}\right)} e^{-i N \theta}
\end{aligned}
$$

The free energy $\mathcal{F}_{F}(\beta \mid N)=-\frac{1}{\beta} \ln \mathcal{Z}_{F}(\beta \mid N)$ therefore consists of two contributions

$$
\begin{gathered}
\mathcal{F}_{F}(\beta \mid N)=\mathcal{F}_{e}(\beta \mid N)-\frac{1}{\beta} \ln \left(\int_{0}^{2 \pi} \Psi(\theta) d \theta\right) . \\
\operatorname{with}_{\mathrm{F}}(\beta \mid \mathrm{N})=-\frac{1}{\beta} \ln \frac{\Xi\left(\mathrm{u}_{\mathrm{e}}, \beta\right)}{\mathrm{u}_{\mathrm{e}}^{\mathrm{N}}},
\end{gathered}
$$

If one would redefine $u_{e}$ as $u_{e}=e^{\beta \mu}$, the relation (19) becomes the common expression for the chemical potential $\mu$ given the total number of particles $N$. However, this expression is a consequence of the mathematical technique used for the inversion of the generating function, and it leads to an approximation for the partition function.

It is well known that the steepest descent method often gives good results in the case of inverse Laplace transforms, but several suggestions are found in the literature
24, 37,38,39,40 to use this method also for this type of inversion of a $Z$ transform. In this case, the approximation turns out to be rather poor, and in general the error estimate poses a problem. Below we shall show that, although the approximation (24) is excellent for fermion systems with completely filled shells in the low temperature limit, it requires important corrections when the shells are not completely filled. It should be emphasized that there is no approximation involved in the use of $u_{e}$ (or any other integration radius $u$ ) as long as only the singularity in the origin is contained in the integration domain. The approximation stems from the replacement of the integrand by its extremum, in the hope that the contribution of the remaining oscillatory contribution to the free energy is negligible.

\subsection{The modified fugacity}

The optimal radius $u_{e}$ for inversion can be calculated from (19) if the energy spectrum of the system is known. After integration over $E$, one obtains the following transcendental equation for $u_{e}$ :

$$
N=\sum_{\nu} g_{\nu} \frac{u_{e} e^{-\beta \epsilon_{\nu}}}{1+u_{e} e^{-\beta \epsilon_{\nu}}}
$$

Assuming the usual relation between the chemical potential and the fugacity $u_{e}=e^{\beta \mu}$ allows in the low temperature limit only solutions that consist of closed shells 4 . In order to have the possibility to incorporate also open shells we assume that $u_{e}$ depends on $\beta$ in the low temperature limit as follows:

$$
u_{e}=\lambda e^{\beta \epsilon_{L}}
$$

where $L$ is the lowest not fully occupied level. Splitting the summation over the levels in the equation for $N$ into three parts we obtain

$$
\begin{aligned}
N & =\sum_{\nu=0}^{L-1} g_{\nu} \frac{\lambda e^{\beta\left(\epsilon_{L}-\epsilon_{\nu}\right)}}{1+\lambda e^{\beta\left(\epsilon_{L}-\epsilon_{\nu}\right)}} \\
& +g_{L} \frac{\lambda}{1+\lambda}+\sum_{\nu=L+1}^{\infty} g_{\nu} \frac{\lambda e^{\beta\left(\epsilon_{L}-\epsilon_{\nu}\right)}}{1+\lambda e^{\beta\left(\epsilon_{L}-\epsilon_{\nu}\right)}}
\end{aligned}
$$

and taking the limit $\beta \rightarrow \infty$ we find the following implicit expression for the parameter $\lambda$ :

$$
N=N_{L}+g_{L} \frac{\lambda}{1+\lambda}
$$

where $N_{L}$ is the total number of states in all fully occupied levels

$$
N_{L}=\sum_{\nu=0}^{L-1} g_{\nu}
$$

This number and therefore the level $L$ itself, has to be determined from

$$
N_{L} \leq N<N_{L+1}
$$


Once $N_{L}$ is known, one can calculate the value of $\lambda$, i.e.

$$
\lambda=\frac{N-N_{L}}{N_{L}+g_{L}-N}=\frac{N-N_{L}}{N_{L+1}-N} .
$$

Note that this calculation explicitly assumed that $N$ differs from $N_{L}$. In the case that $N=N_{L}$, i.e., if the level $L$ is empty, one obtains $u_{e}=e^{\beta \epsilon_{L}}$ in the low temperature limit. It is interesting to remark that the divergent terms in the steepest descent inversion for bosons could be eliminated by Landsberg using a similar modification of the fugacity [5].

Using now the generating function $\Xi\left(\beta, u_{e}\right)$ in the limit $\beta \rightarrow \infty$ with $u_{e}=\lambda e^{\beta \epsilon_{L}}$, one obtains the following zero-order approximation $\mathcal{Z}_{e}(\beta \mid N)$ for the partition function

$$
\lim _{\beta \rightarrow \infty} \mathcal{Z}_{e}(\beta \mid N)=\frac{e^{-\beta E_{0}}\left(N_{L+1}-N_{L}\right)^{N_{L+1}-N_{L}}}{\left(N-N_{L}\right)^{N-N_{L}}\left(N_{L+1}-N\right)^{N_{L+1}-N}}
$$

with the fermion ground state energy $E_{0}$ given by

$$
E_{0}=\sum_{\nu=0}^{L-1} g_{\nu} \epsilon_{\nu}+\left(N-N_{L}\right) \epsilon_{L}
$$

The integral correction $\int_{0}^{2 \pi} \Psi(\theta) d \theta$ to the partition function can be derived from

$$
\lim _{\beta \rightarrow \infty} \Psi(\theta)=\frac{1}{2 \pi}\left(\frac{1+\lambda e^{i \theta}}{1+\lambda}\right)^{g_{L}} e^{-i \theta\left(N-N_{L}\right)},
$$

and hence, using the binomial expansion of $\left(1+\lambda e^{i \theta}\right)^{g_{L}}$, one obtains

$$
\begin{aligned}
\lim _{\beta \rightarrow \infty} \int_{0}^{2 \pi} \Psi(\theta) d \theta & =\left(\begin{array}{c}
N_{L+1}-N_{L} \\
N-N_{L}
\end{array}\right)\left(N-N_{L}\right)^{N-N} \\
& \times \frac{\left(N_{L+1}-N\right)^{N_{L+1}-N}}{\left(N_{L+1}-N_{L}\right)^{N_{L+1}-N_{L}}}
\end{aligned}
$$

The partition function thus becomes

$$
\lim _{\beta \rightarrow \infty} \mathcal{Z}_{F}(\beta \mid N) \rightarrow\left(\begin{array}{c}
g_{L} \\
N-N_{L}
\end{array}\right) e^{-\beta E_{0}},
$$

where the combinatorial factor in front accounts for the number of possibilities to occupy the open shell. In order to obtain this factor, the modification of the fugacity is not sufficient: the inversion procedure has to be carried out completely. In other words: the open shell partition function of fermions in the low temperature limit has to be calculated by an inversion because the contribution of the integral correction turns out to be crucial!

But the exact ground state energy (33) is obtained from the approximate expression (24). This means that the integral correction to the free energy (23) becomes negligible in the low temperature limit; it only contributes to the degeneracy factor in the partition function, i.e., to the entropy. In the next section we will investigate whether this coincidence also remains valid for the correlation functions.

\section{The density of an open shell Fermion gas}

In this section we derive the one-point correlation function that describes the density in the ground-state, using the exact inversion. Furthermore, we show that the use of the modified fugacity recovers the exact result.

\subsection{The density and its generating function}

The generating function for the Fourier transform $\tilde{n}_{\mathbf{q}}$ of the density of a $N$-fermion system has been derived in 14. It is given by

$$
\mathcal{G}_{\tilde{n}_{\mathbf{q}}}(\beta, u)=\sum_{N=0}^{\infty} \tilde{n}_{\mathbf{q}}(\beta, N) \mathcal{Z}_{F}(\beta \mid N) N u^{N}
$$

and it can easily be expressed in terms of the density of states

$$
\frac{\mathcal{G}_{\tilde{n}_{\mathbf{q}}}(\beta, u)}{\Xi_{F}(\beta, u)}=\int f_{E}(u, \beta) \Omega(E, \mathbf{r}) e^{-i \mathbf{q} \cdot \mathbf{r}} d \mathbf{r} d E,
$$

where $f_{E}(u, \beta)$ has the form of an occupation function, but $u$ is still the auxiliary variable to construct the power series

$$
f_{E}(u, \beta)=\frac{u e^{-\beta E}}{1+u e^{-\beta E}}
$$

\subsection{The exact inversion}

Inverting (37) for $\tilde{n}_{\mathbf{q}}(\beta, N)$ by the contour integral technique, and filling out $\mathcal{G}_{\tilde{n}_{\mathbf{q}}}(\beta, z)$ in the form (38)

$$
\begin{aligned}
\tilde{n}_{\mathbf{q}}(\beta, N) & =\frac{1}{N} \frac{1}{\mathcal{Z}_{F}(\beta \mid N)} \sum_{\nu=0}^{\infty} n_{\nu}(\mathbf{q}) \frac{1}{2 \pi i} \\
& \times \lim _{u \rightarrow 0} \oint_{|z|=u} \Xi_{F}(\beta, z) \frac{z e^{-\beta \epsilon_{\nu}}}{1+z e^{-\beta \epsilon_{\nu}}} \frac{d z}{z^{N+1}},
\end{aligned}
$$

the contour integral can be transformed into an angular integral. One obtains after some effort

$$
\lim _{\beta \rightarrow \infty} \tilde{n}_{\mathbf{q}}(\beta, N) \rightarrow \frac{1}{N}\left(\sum_{\nu=0}^{L-1} n_{\nu}(\mathbf{q})+\frac{N-N_{L}}{N_{L+1}-N_{L}} n_{L}(\mathbf{q})\right),
$$

with $n_{\nu}(\mathbf{q})$ is defined as the Fourier transform of the density contribution from the single-particle wave functions in the level $\nu$

$$
n_{\nu}(\mathbf{q})=\sum_{k=1}^{g_{\nu}} \int\left|\varphi_{\nu, k}(\mathbf{r})\right|^{2} e^{-i \mathbf{q} \cdot \mathbf{r}} d \mathbf{r} .
$$

This result for the density could have been guessed by realizing that for an open shell the states belonging to that shell must have the same probability of being occupied. 


\subsection{The optimal fugacity}

The generating function for the density in the spectral representation (4) can be written as

$$
\frac{\mathcal{G}_{\tilde{n}_{\mathbf{q}}}(\beta, u)}{\Xi_{F}(\beta, u)}=\sum_{\nu=0}^{\infty} \frac{u e^{-\beta \epsilon_{\nu}}}{1+u e^{-\beta \epsilon_{\nu}}} n_{\nu}(\mathbf{q})
$$

Repeating the approximate inversion as for the partition function from the previous section, using again

$$
u_{e}=\lambda e^{\beta \epsilon_{L}},
$$

one finds

$$
\lim _{\beta \rightarrow \infty} \frac{\mathcal{G}_{\tilde{n}_{\mathbf{q}}}\left(\beta, u_{e}\right)}{\Xi_{F}\left(\beta, u_{e}\right)}=\sum_{\nu=0}^{L-1} n_{\nu}(\mathbf{q})+\frac{N-N_{L}}{N_{L+1}-N_{L}} n_{L}(\mathbf{q}) .
$$

The modified fugacity thus allows to calculate the groundstate density exactly, similarly as we found for the groundstate energy.

\section{4 $\mathrm{A}$ useful relation}

Because the generating function often can be written in closed form, the following exact relation

$$
\lim _{\beta \rightarrow \infty} \tilde{n}_{\mathbf{q}}(\beta, N)=\frac{1}{N} \lim _{\beta \rightarrow \infty} \frac{\mathcal{G}_{\tilde{n}_{\mathbf{q}}}\left(\beta, u_{e}\right)}{\Xi_{F}\left(\beta, u_{e}\right)},
$$

between the results for the ground-state of the two inversion methods turns out to be of practical numerical importance. The density, i.e. the sum of single-particle contributions over all the occupied states, can be obtained from an appropriate limit of a ratio of two generating functions. For fermions in a harmonic potential $\mathcal{G}_{\tilde{n}_{\mathbf{q}}}(\beta, u) / \Xi_{F}(\beta, u)$ is known in closed form and the limit can be used to obtain an explicit result for the density, thus avoiding a tedious summation over occupied states.

\section{The polarization function of an open shell fermion gas}

In this section we investigate whether the two-point correlation function that describes the density-density correlations of the fermion system can also be obtained in the low temperature limit using a modified chemical potential. Unfortunately, this is not the case. The exact inversion of the generating function and the use of the chemical potential give analogous but slightly different results. Both calculations use the same techniques as explained in the preceding sections. Therefore only an outline of the method and a summary of the results will be given.

The Fourier transform of the two-point correlation function is defined as:

$$
N^{2} \tilde{C}_{\mathbf{q}^{\prime}, \mathbf{q}}(\tau \mid \beta, N)=\left\langle\sum_{j=1}^{N} \sum_{l=1}^{N} e^{-i \mathbf{q}^{\prime} \cdot \mathbf{r}_{j}^{\prime}(\tau)} e^{-i \mathbf{q} \cdot \mathbf{r}_{l}(0)}\right\rangle
$$

An outline of the derivation of this function can be found in 41. Defining the following generating function for the two point correlation function

$$
G_{\tilde{C}_{\mathbf{q}^{\prime}, \mathbf{q}}}(\tau \mid \beta, u)=\sum_{N=0}^{\infty} \tilde{C}_{\mathbf{q}^{\prime}, \mathbf{q}}(\tau \mid \beta, N) \mathcal{Z}_{F}(\beta \mid N) N^{2} u^{N}
$$

a straightforward application of the techniques illustrated in our former publications 13 14 leads after a lengthy but straightforward calculation to an expression obtained by performing the contour integral of the inversion exactly in the limit $\beta \rightarrow \infty$ :

$$
\begin{aligned}
\lim _{\beta \rightarrow \infty} N^{2} \tilde{C}_{\mathbf{q}^{\prime}, \mathbf{q}}(\tau \mid \beta, N) & =A_{0}\left(\mathbf{q}, \mathbf{q}^{\prime}, \tau\right) \\
& +\frac{N-N_{L}}{N_{L+1}-N_{L}} A_{1}\left(\mathbf{q}, \mathbf{q}^{\prime}, \tau\right) \\
& +\frac{N-N_{L}}{N_{L+1}-N_{L}} \frac{N-N_{L}-1}{N_{L+1}-N_{L}-1} \\
& \times A_{2}\left(\mathbf{q}, \mathbf{q}^{\prime}, \tau\right)
\end{aligned}
$$

with

$$
\begin{aligned}
A_{0}\left(\mathbf{q}, \mathbf{q}^{\prime}, \tau\right)= & \sum_{\nu=0}^{L-1} n_{\nu}(\mathbf{q}) \sum_{\nu^{\prime}=0}^{L-1} n_{\nu^{\prime}}\left(\mathbf{q}^{\prime}\right) \\
+ & \sum_{\nu=0}^{L-1} \sum_{\nu^{\prime}=L}^{\infty} e^{\tau\left(\epsilon_{\nu}-\epsilon_{\nu^{\prime}}\right)} M_{\nu, \nu^{\prime}}\left(\mathbf{q}, \mathbf{q}^{\prime}\right), \\
A_{1}\left(\mathbf{q}, \mathbf{q}^{\prime}, \tau\right)= & n_{L}(\mathbf{q}) \sum_{\nu=0}^{L-1} n_{\nu}\left(\mathbf{q}^{\prime}\right) \\
+ & n_{L}\left(\mathbf{q}^{\prime}\right) \sum_{\nu=0}^{L-1} n_{\nu}(\mathbf{q}) \\
& +\sum_{\nu=L}^{\infty} e^{\tau\left(\epsilon_{L}-\epsilon_{\nu}\right)} M_{L, \nu}\left(\mathbf{q}, \mathbf{q}^{\prime}\right) \\
& -\sum_{\nu=0}^{L-1} e^{\tau\left(\epsilon_{\nu}-\epsilon_{L}\right)} M_{\nu, L}\left(\mathbf{q}, \mathbf{q}^{\prime}\right) \\
A_{2}\left(\mathbf{q}, \mathbf{q}^{\prime}, \tau\right)= & n_{L}(\mathbf{q}) n_{L}\left(\mathbf{q}^{\prime}\right)-M_{L, L}\left(\mathbf{q}, \mathbf{q}^{\prime}\right)
\end{aligned}
$$

and

$$
\begin{aligned}
& M_{\nu, \nu^{\prime}}\left(\mathbf{q}, \mathbf{q}^{\prime}\right)=\sum_{k=1}^{g_{\nu}} \sum_{k^{\prime}=1}^{g_{\nu^{\prime}}} \Lambda_{\nu, k ; \nu^{\prime}, k^{\prime}}(\mathbf{q}) \Lambda_{\nu^{\prime}, k^{\prime} ; \nu, k}\left(\mathbf{q}^{\prime}\right) \\
& \Lambda_{\nu, k ; \nu^{\prime}, k^{\prime}}(\mathbf{q})=\int \varphi_{\nu, k}^{*}(\mathbf{r}) \varphi_{\nu^{\prime}, k^{\prime}}(\mathbf{r}) e^{-i \mathbf{q} \cdot \mathbf{r}} d \mathbf{r}
\end{aligned}
$$

It should be noted that the expression for $A_{2}\left(\mathbf{q}, \mathbf{q}^{\prime}, \tau\right)$ implies that this term is independent of Euclidean time $\tau$.

It turns out that the result (48) is not obtained with the approximate inversion $G_{\tilde{C}_{\mathbf{q}^{\prime}, \mathbf{q}}}\left(\tau \mid \beta, u_{e}\right) / \Xi_{F}\left(\beta, u_{e}\right)$ of the generating function. Using again $u_{e}=\lambda e^{\beta \epsilon_{L}}$ the dominant terms for $\beta \rightarrow \infty$ instead become

$$
\lim _{\beta \rightarrow \infty} \frac{G_{\tilde{C}_{\mathbf{q}^{\prime}, \mathbf{q}}}\left(\tau \mid \beta, u_{s d}\right)}{\Xi_{F}\left(\beta, u_{s d}\right)}=A_{0}\left(\mathbf{q}, \mathbf{q}^{\prime}, \tau\right)
$$




$$
\begin{aligned}
& +\frac{N-N_{L}}{N_{L+1}-N_{L}} A_{1}\left(\mathbf{q}, \mathbf{q}^{\prime}, \tau\right) \\
& +\frac{N-N_{L}}{N_{L+1}-N_{L}} \frac{N-N_{L}}{N_{L+1}-N_{L}} \\
& \times A_{2}\left(\mathbf{q}, \mathbf{q}^{\prime}, \tau\right) .
\end{aligned}
$$

Although very similar to the exact result (48) the combinatorial factors for the not fully occupied level are slightly different. The justification of this approximate inversion is rather elaborate. E.g., it is not trivial that the contour integration around the origin can be extended to the large radius $u_{e}$ without introducing extra poles. This point has been carefully checked.

Although very similar to the exact result (48) the combinatorial factors in (55) for the not fully occupied level are not correct. Therefore the similar correspondence as found for the partition function and for the density (45), is valid for the two-point correlation function in the case of closed shells only. Indeed, $A_{1}\left(\mathbf{q}, \mathbf{q}^{\prime}, \tau\right)$ and $A_{2}\left(\mathbf{q}, \mathbf{q}^{\prime}, \tau\right)$ do not contribute to the polarization function for the case of closed shells, i.e., for $N=N_{L}$. This leads to:

$$
\lim _{\beta \rightarrow \infty} N^{2} \tilde{C}_{\mathbf{q}^{\prime}, \mathbf{q}}(\tau \mid \beta, N)=\lim _{\beta \rightarrow \infty} \frac{G_{\tilde{C}_{\mathbf{q}^{\prime}, \mathbf{q}}}\left(\tau \mid \beta, u_{e}\right)}{\Xi\left(\beta, u_{e}\right)} .
$$

\section{Discussion and Conclusions}

Several authors suggested or stated that calculations performed with the methods developed in the grand canonical ensemble cannot be trusted when one deals with a given fi-

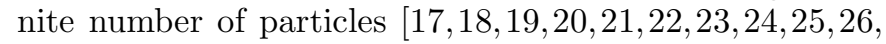
27. In this paper we have pinpointed the origin of this discrepancy for open shell Fermion systems. Our analysis is based on an exact inversion of the existing transform between the partition function, the one-point correlation function and the two point-correlation function for $N$ particles and their generating functions. We observed that the generating function of the partition function for the proper parameter choice becomes the grand canonical partition function of the same model. This observation allows to compare the results for the exact inversion with those relying on the chemical potential. It should be stressed that a modification of the relation between the fugacity and the chemical potential is needed to obtain the correct ground state energy, but this modification is not sufficient to obtain the low temperature limit of the partition function correctly in the case of open shell fermion gases: the entropy requires the exact inversion. In summary the modification of the fugacity definition for open shells leads to the correct expressions for the ground-state energy and the ground-state density. But it is not good enough to obtain the low temperature limit of the partition function and the polarization function, and a correction is needed, as derived above. This situation is summarized in table 1 .

Note that the differences discussed here have their origin in the degeneracy of the lowest not completely occupied level. In the absence of this degeneracy, i.e. for
Table 1. This table summarizes for which quantities and type of systems the use of the unmodified chemical potential $\mu$ and of its modified form leads to correct results.

\begin{tabular}{lll}
\hline Quantity & $\mu$ & Modified $\mu$ \\
\hline Ground state energy & closed shell & open shell \\
Density & closed shell & open shell \\
Partition function & closed shell & extra correction \\
Correlation function & closed shell & extra correction \\
\hline
\end{tabular}

closed shell systems, the ground-state expressions of both inversion schemes are identical. But if there are $N-N_{L}$ fermions in the lowest not completely occupied level, a correction term proportional to

$$
\frac{N-N_{L}}{N_{L+1}-N_{L}}
$$

is introduced in the ground-state energy and the density by modifying the fugacity. An extra term proportional to

$$
\frac{\left(N-N_{L}\right)\left(N-N_{L}-1\right)}{\left(N_{L+1}-N_{L}\right)\left(N_{L+1}-N_{L}-1\right)}
$$

is found for the polarization function.

The relation between the density of states, the partition function and the generating function have been discussed by several authors. In classical statistical mechanics they are attributed to Fowler and Darwin 42 43. In quantum statistical mechanics they are discussed in the book by P.T. Landsberg [37] who makes an epistemological analysis of them (see, e.g., ref. 44]). A mathematical justification and review of the present understanding can be found in [45]. Of course the present findings also oblige to scrutinize the use of the chemical potential in other calculation schemes like the density functional approach or the Gross-Pitaevsky equation. The questions are there: "How can one control these methods, clearly based on the grand canonical ensemble, on their correctness for a finite number of particles?", and "Is a modification of the chemical potential in these cases also necessary?". Our preliminary investigations indicate that in the case that the levels are degenerate, one should use the modified chemical potential in order to get the energy and the density correct, and to obtain a fair approximation for the two-point correlation function.

This work is performed within the framework of the FWO projects No. 1.5.545.98, G.0287.95, G.0071.98,

and WO.073.94N [Wetenschappelijke Onderzoeksgemeenschap van het FWO over "Laagdimensionele systemen" (Scientific Research Community on Low-Dimensional Systems)], the "Interuniversitaire Attractiepolen - Belgische Staat, Diensten van de Eerste Minister - Wetenschappelijke, Technische en Culturele Aangelegenheden" (Interuniversity Poles of Attraction Programs -Belgian State, Prime Minister's Office -Federal Office for Scientific, Technical and Cultural Affairs), and in the framework of the GOA BOF UA 2000 projects of the Universiteit Antwerpen. 


\section{References}

1. A. A. Abrikosov, L. P. Gorkov, I. E. Dzyaloshinski, Methods of Quantum Field Theory in Statistical Physics, (Pergamon, Oxford, 1965).

2. A. L. Fetter and J. D. Walecka, Quantum Theory of Many Particle Systems, (Mc Graw-Hill, New-York, 1971).

3. J. W. Negele and H. Orland, Quantum Many-Particle Systems, (Perseus books, Reading, 1998).

4. J. Schneider and H. Wallis, Phys. Rev. A 57, 1253 (1998).

5. P. Landsberg Phys. Rev. 94, 469 (1954).

6. R. Kubo, Statistical Mechanics, (North-Holland, Amsterdam, 1974).

7. R. P. Feynman, Statistical Mechanics, a Set of Lectures, (W. A. Benjamin Inc., Reading, 1972).

8. R. P. Feynman, Phys. Rev. 91, 1291 (1953).

9. T. Matsubara, Prog. Theor. Phys. 6, 714 (1951).

10. O. Penrose and L. Onsager, Phys. Rev. 104, 576 (1956).

11. A. P. Lyubartsev and P. Vorontsov-Velyaminov, Phys. Rev. A 48, 4075 (1993).

12. D. M. Ceperley, Rev. Mod. Phys. 67, 279 (1995).

13. F. Brosens, J. T. Devreese, and L. F. Lemmens, Phys. Rev. E 55, 227 (1997); E 55, 6795 (1997).

14. F. Brosens, J. T. Devreese, and L. F. Lemmens, Phys. Rev. E 57, 3871 (1998); E 58, 1634 (1998).

15. P. N. Vorontsov-Velyaminov, S. D. Ivanov, and R. I. Gorbunov, Phys. Rev. E 59, 168 (1999).

16. H.-T. Elze and W. Greiner, Phys. Rev. A 33, 1879 (1986).

17. R. M. Ziff, G. E. Uhlenbeck, and M. Kac, Phys. Rep. 32, 169 (1977).

18. I. Fujiwara, D. ter Haar, and H. Wergeland, J. Stat. Phys. 2, 329 (1970).

19. F. Brosens, J. T. Devreese, and L. F. Lemmens, Solid State Commun. 100, 123 (1996).

20. S. Grossmann and M. Holthaus, Phys. Rev. Lett. 79, 3557 (1997).

21. P. Navez, D. Bitouk, M. Gajda, Z. Idziaszek, and K. Rzążewski, Phys. Rev. Lett. 79, 1789 (1997).

22. M. Gajda and K. Rzążewski, Phys. Rev. Lett. 78, 2686 (1997).

23. Z. Idziaszek, M. Gajda, P. Navez, M. Wilkens, and K. Rzążewski, Phys. Rev. Lett 82, 4376 (1999).

24. M. Holthaus and E. Kalinowski, Annals of Physics 267, 321 (1999); arXiv:cond-matt/9906092 (1999).

25. R. Rossignoli, Phys. Rev. C 51, 1772 (1995).

26. S. Pratt, Phys. Rev. Lett. 84, 4255 (2000).

27. V. Meden and K. Schonhammer, Phys. Rev. Lett. 85, 5254 (2000).

28. L. F. Lemmens, F. Brosens, and J. T. Devreese, Solid State Commun. 109, 615 (1999); arXiv:cond-matt/9803168 (1998).

29. P. Whittle, Probability, (Penguin Books, Middlesex, 1970).

30. L. F. Lemmens, F. Brosens, and J. T. Devreese, Phys. Rev. E 61, 3358 (2000).

31. J. Ginibre, J. Math. Phys. 6, 238 (1965); 6, 252 (1965); 6, 1432 (1965).

32. J. Ginibre in Statistical Mechanics and Quantum Field Theory, 1971, Les Houches Lectures, edited by C. de Witt and R. Stora, (Gordon and Breach, New York, 1971).

33. A. Z. Mekjian and S. J. Lee, Phys. Rev. A 44, 6294 (1991).

34. K. C. Chase and A. Z. Mekjian, Phys. Rev. C 50, 2078 (1994).

35. H. Sato, Phys. Rev. C 36, 785 (1987).
36. P. Borrmann and G. Franke, J. Chem. Phys. 98, 2484 (1993).

37. P. T. Landsberg, Thermodynamics with quantum statistical illustrations, (Interscience Publishers, New York, London, 1961).

38. E. Schrödinger, Statistical Thermodynamics,(Cambridge University Press, Cambridge, 1946).

39. R. B. Dingle, Proc. Cambridge Phil. Soc. 45, 275 (1949).

40. R. B. Dingle, Asymptotic Expansions: Their Derivation and Interpretation, (Academic Press, New York and London, 1973; chap. XI).

41. L. F. Lemmens, D. Saeys, F. Brosens, and J. T. Devreese, Solid State Comm 117, 357 (2001).

42. C. G. Darwin, R. H. Fowler, On the partition of energy, Phil. Mag. 44, 450 (1922).

43. C. G. Darwin and R. H. Fowler, Proc. Cambridge Phil. Soc. 21, 391, 730 (1923).

44. H. Schmidt and J. Schnack, arXiv:cond-mat/0104293 Am. J. Phys. 70, 53 (2002).

45. V. Kostrykin and R. Schrader, arXiv:cond-mat/0109034 (2001), Mathematical Results in Quantum Mechanics R. Weder, P. Exner, and B. Grebert (Eds.), Contemporary Mathematics 307 , Amer. Math. Soc., 177 (2002) 